\title{
El profesorado de Educación Secundaria Obligatoria y Bachillerato ante la Diversidad Sexual
}

Vanesa Romero Códez. Universidad de Granada

Miguel Ángel Gallardo Vigil. Universidad de Granada

Recepción: 29.06.2019 | Aceptado: 30.10.2019

Correspondencia a través de ORCID: Miguel Ángel Gallardo Vigil

D 0000-0002-5462-077X

Citar: Romero, V. y Gallardo, MA. (2019). El profesorado de Educación Secundaria Obligatoria y Bachillerato ante la Diversidad Sexual, REIDOCREA, 8, 357-366.

Resumen: A pesar de los avances legislativos y los cambios sociales, todavía siguen existiendo actitudes homófobas hacia las personas lesbianas, gais, bisexuales, transexuales e intersexuales. Una de las vías para acabar con esta discriminación reside en los centros educativos y en especial en el profesorado. Este estudio pretende conocer el grado de prejuicio y distancia social que los profesores de secundaria tienen hacia las personas con diversidad sexual y también evaluar la formación o falta de esta en torno a esta temática. A través de un estudio de carácter cuantitativo y utilizando un cuestionario, se ha recogido información en torno a estos temas. Los datos nos muestran que sigue existiendo una parte del profesorado con alta carga de prejuicios hacia los gais y lesbianas, así como de distancia social. Variables como el sexo, la religión o la ideología política son moderadoras de esta carga de prejuicios. Es importante que, en la formación inicial de los docentes, así como en la formación de posgrado, se incluya la diversidad sexual como un tema relevante y necesario que podrá favorecer la posterior aplicación en el aula y evitar conductas de acoso entre los estudiantes por la condición sexual.

Palabras clave: Diversidad Sexual | Profesorado

Teachers of compulsory secondary education and baccalaureate about sexual diversity

Abstract: Despite legislative advances and social changes, homophobic attitudes toward lesbian, gay, bisexual, transgender and intersex persons still exist. One of the ways to end this discrimination resides in the educational centers and especially in the teaching staff. This study aims to know the degree of prejudice and social distance that secondary school teachers have towards people with sexual diversity and also to evaluate the training or lack thereof in relation to this topic. Through a quantitative study and using a questionnaire, information has been gathered around these topics. The data shows that there is still a part of the teaching staff with a high burden of prejudice towards gays and lesbians, as well as social distance. Variables such as sex, religion or political ideology are moderating this burden of prejudice. It is important that, in the initial training of teachers, as well as in postgraduate training, sexual diversity be included as a relevant and necessary topic that may favor subsequent application in the classroom and avoid harassment behavior among students by the sexual condition.

Keywords: Sexual Diversity | teachers

\section{Introducción}

En el año 2018 se celebró en España el 40 aniversario de la primera manifestación organizada por parte de los colectivos de gais, lesbianas y transexuales. Esta primera marcha realizada en 1978 en Madrid, congregó a más de 7000 personas que salieron a la calle pidiendo un cambio legislativo que dejara de considerar como delito determinadas opciones sexuales.

Desde aquella primera manifestación hasta la fecha, se han ido dando pasos que favorecen la visibilidad del colectivo de lesbianas, gais, transexuales, bisexuales e intersexuales (LGTBI) así como la adquisición de diversos derechos, si bien, todavía encontramos actitudes negativas hacia este colectivo.

España, es el tercer país que aprueba la unión entre personas del mismo sexo, solo por detrás de Países Bajos y Bélgica. Así, en el Boletín oficial del Estado, la Ley 13/2005, 
de 1 de julio por la que se modifica el Código Civil en materia de derecho a contraer matrimonio, recoge en su artículo 44 que "El matrimonio tendrá los mismos requisitos y efectos cuando ambos contrayentes sean del mismo o de diferente sexo" (p. 23633).

Dos años más tarde, a través de la Ley 3/2007, de 15 de marzo, reguladora de la rectificación registral de la mención relativa al sexo de las personas, permite rectificar en el registro el sexo de una persona, favoreciendo el cambio del propio nombre de la persona de tal forma que no resulte discordante con su sexo en el registro.

El estudio realizado por Pew Reserch Center (2013), afirma que el 88\% de los españoles están de acuerdo con que la sociedad debería aceptar la homosexualidad y solo un $11 \%$ estaría en contra de esta afirmación. España se convierte así en el primer país del mundo con mayor aceptación de los homosexuales, seguida de Alemania con un $87 \%$ a favor y de Canadá o República Checa con un $80 \%$. En el lado opuesto se sitúa Nigeria con un $98 \%$ en contra de la homosexualidad seguida de Jordania con un $97 \%$.

No obstante, y a pesar de que la propia legislación ha avanzado en derecho de las personas LGTBI, de la opinión que la mayoría de la sociedad española tiene, se siguen dando situaciones de LGTBIfobia. Las actitudes negativas hacia estas personas, seguimos encontrando

situaciones de rechazo que provocan una distancia social en miembros del colectivo, y ponen de manifiesto que la legislación en materia de igualdad, al igual que sucede con la violencia de género, necesita nuevos mecanismos para promover la reeducación social, partiendo de los ámbitos familiar y educativo, ya que son los entornos más inmediatos donde se desarrollan los primeros prejuicios. (Alemán-Calvache, 2018, p. 6)

De hecho, diversos estudios afirman que los jóvenes LGTBI son más propensos al suicidio, entre 1,5 y 3 veces más en el caso de haberlo intentado, que sus pares no LGTBI (Russell, 2013), sufren una opresión heterosexista (Chan y Erby, 2018), o en algunos países, reciben sanciones religiosas y morales (Vázquez-Rivera, Caleb y Toro Alfonso, 2018). Además, como indica Méndez (2007), en nuestras aulas se sigue dando bullying homofóbico hacia aquellos niños y jóvenes que no se encuentran dentro de los cánones de la heteronormatividad; y, como indica Pichardo y De Stefano (2015), la homofobia es uno de los principales motivos de acoso escolar. Byne (1994), en multitud de ocasiones el profesorado no detecta este acoso confundiendo este hecho con vandalismo, y como indican Francisco Amat, Aguirre García-Carpintero y Moliner Miravet (2018) esto "contrasta con la desinformación que tienen y la falta de contenidos tanto en las carreras que han estudiado como el máster que les capacita para la función docente" (p. 95).

El avance de la extrema derecha en Europa y en otros países, con un discurso del odio, está calando en la sociedad y se está fomentando un retroceso en los derechos ya adquiridos por el colectivo LGTBI.

Todo esto está incidiendo en la existencia de una discriminación directa e indirecta hacia las personas que no cumplen con la heterormatividad, lo que también se ve influenciado por una falta de conocimientos e información sobre la diversidad sexual.

Diversas variables son moderadoras de estas actitudes negativas.

- Sexo: diversos estudios demuestran que las personas de sexo masculino presentan una mayor actitud negativa que las de sexo femenino (Gallardo-Vigil, Segura-Robles y García-Alonso, 2017; Jäckle y Wenzelburger, 2015; Robles- 
Reina, Alemany-Arrebola y Gallardo-Vigil, 2017; Rodríguez Castro y Alonso Ruido, 2017; Rodríguez Otero y Treviño Martínez, 2016).

- Religión y práctica religiosa: en referencia a la religión y su práctica, la literatura indica que también tienen influencia en las actitudes hacia las personas LGTBI. Así pues, aquellas personas con fuerte práctica religiosa presentan prejuicios más negativos (López y Taype-Roldán, 2017; Saraç, 2015). Por el contrario, los no creyentes son los que muestran actitudes más positivas.

- Ideología política: aquellas personas que se identifican con posiciones políticas conservadoras presentan mayores prejuicios hacia las personas LGTBI (Anderson, Koc y Farlomir-Pichastor, 2018; Gallardo-Vigil y Rodríguez-Serrano, 2008).

Como educadores, debemos evitar la homofobia dentro y fuera del aula. La información y la formación de los jóvenes y docentes en temas de diversidad sexual será una apuesta decidida por la inclusión de todas las personas con independencia de su orientación sexual e identidad de género y favorecerá, por tanto, la tolerancia y la visibilidad de un colectivo que, en multitud de ocasiones (aunque cada vez sea menos), se siente discriminado y oculto. Es imprescindible, como indican Gallardo-Vigil, RojasRuiz y López Vallejo (2019), "la introducción de los diferentes aspectos del desarrollo afectivo-sexual en el currículum académico, desde las etapas educativas iniciales, hasta la enseñanza superior" (p. 84).

\section{Objetivos}

\section{Objetivo general}

Este objetivo de esta investigación reside, por tanto, en la necesidad de conocer la percepción que presenta el profesorado de Educación Secundaria Obligatoria y Bachillerato acerca de la diversidad sexual y comprobar si hay factores como la ideología política, el género o las creencias religiosas, entre otros, que puedan influir en esta percepción.

\section{Objetivos específicos}

- Conocer el grado de prejuicio hacia las personas con diversa orientación sexual e identidad de género que tiene el profesorado de Educación Secundaria Obligatoria y Bachillerato.

- Determinar el grado de distancia social que presenta el profesorado de Educación Secundaria Obligatoria y Bachillerato hacia las personas LGTBI.

- Evaluar el grado de interés y formación acerca de la diversidad sexual por parte del profesorado de Educación Secundaria Obligatoria y Bachillerato.

\section{Métodos}

Para el desarrollo de esta investigación se ha seguido una metodología empíricoanalítica. Se trata de un estudio ex post-facto de tipo correlacional.

\section{Participantes}

Los participantes del estudio han sido los profesores de Educación Secundaria Obligatoria y Bachillerato de un Instituto de Educación Secundaria Obligatoria de la Ciudad Autónoma de Melilla. Se invitó al total del claustro docente $(\mathrm{N}=60)$, obteniendo una muestra productora de datos de $n=35$, lo que supone el $58,3 \%$ del total. Los participantes presentan una edad que oscila entre los 25 y los 58 años, siendo la edad 
media de 41.06 años. En cuanto al sexo, el $49 \%$ son mujeres frente al 51 que son hombres.

En referencia a la religión y su práctica, casi un $23 \%$ afirma no ser creyente. De los que se han identificado con alguna religión el $37.1 \%$ se define como poco practicante, el $31.4 \%$ como bastante practicante y un $11.4 \%$ como nada practicante.

En cuanto a la ideología política, el $34.3 \%$ se define de centro, seguido de los de izquierdas con un $31.4 \%$. Por su parte, solo el $2.9 \%$ afirma ser de derechas. En los puntos intermedios se encuentran los de centro izquierda con un $11,4 \%$ y los de centro derecha con un $17.1 \%$.

\section{Instrumentos}

Para la recogida de datos de las distintas variables de estudio, se ha utilizado un cuestionario conformado por cuatro partes. La parte primera se centra en recoger la información sobre las variables sociodemográficas de los participantes, a saber, sexo, edad, religión, práctica religiosa e ideología política. Para la segunda parte, centrada en analizar el grado de prejuicios, se utilizó la Escala de prejuicios hacia personas gais y lesbianas, de Toro-Alonso y Varas-Díaz (2004). Para conocer el grado de distancia social que presentan los encuestados, se incluyó como tercer parte del cuestionario la Escala de Distancia Social de gais y lesbianas desarrollada por García (1984). Finalmente, el cuestionario incluyó varias preguntas con la intención de poder determinar si el profesorado era consciente de la existencia de homofobia en los centros educativos, así como su consideración sobre si los estudiantes estarían interesados en la inclusión de temas relacionados con la diversidad afectivo-sexual y el nivel de formación e interés en la formación, por parte del profesorado, en esta temática.

En cuanto a la Escala de prejuicios hacia personas gais y lesbianas, está formada por un total de 46 reactivos que miden el grado de actitudes prejuiciosas hacia las personas con esta orientación sexual. Es una escala tipo Likert con 4 puntos con valores que van desde totalmente de acuerdo a totalmente en desacuerdo. La puntuación que se puede obtener en esta escala oscila entre 46 puntos y 184 puntos, siendo el valor más alto un indicativo de mayor grado de prejuicio. Para la corrección de la escala hay que tener en cuenta que hay un total de 20 ítems que deben ser invertidos para el cálculo de la puntuación global para cada sujeto participante. Esta escala cuenta con cuatro niveles en función de la puntuación total obtenida: de 46 a 56 nivel muy bajo de prejuicio; de 57 a 70 indica un nivel bajo; de 71 a 87 es un nivel alto; y, de 88 a 184 nivel muy alto de prejuicio. La versión original de la escala obtuvo un alfa de Cronbach de 0.91 (ToroAlonso y Varas-Díaz, 2004).

Por su parte, para la Escala de Distancia Social de gais y lesbianas, está formada por 10 reactivos. Las valoraciones oscilan entre 1=viviría con ellos, $2=$ los/as aceptaría como amigos, 3=preferiría tenerlos/as como conocidos, 4=los/as excluiría de mis relaciones sociales y $5=$ no quisiera ni verlos. La puntuación que se puede obtener va desde los 10 puntos hasta los 50 y como en la escala anterior, a mayor puntuación mayor la distancia social hacia gais y lesbianas. También cuenta con 4 niveles: de 10 a 20 baja o ninguna distancia social; de 21 a 30 alguna distancia social; de 31 a 40 distancia social moderada; y, de 41 a 50 alta distancia social hacia gais y lesbianas. Cuenta con un alfa de Cronbach de 0.93 (Toro-Alonso y Varas-Díaz, 2004). 


\section{Procedimiento}

Para la realización de este estudio se solicitó, a través de un proyecto de investigación, autorización a la Dirección Provincial del Ministerio de Educación y Formación Profesional en Melilla. Una vez concedido el permiso, se contactó con la dirección del centro en el que se iba a realizar la recogida de datos. A partir de ese momento se contactó con todos los docentes del IES y se informó de en qué consistía el estudio, así como del anonimato de las respuestas. Tras dos semanas se pasó por el centro para la recogida de todos los cuestionarios cumplimentados.

Análisis de datos

Para llevar a cabo el análisis de los datos se ha utilizado el paquete estadístico SPSS en su versión 20.0 y se han realizado análisis estadísticos descriptivos e inferenciales con la intención de poder dar respuesta a los objetivos planteados en el estudio.

\section{Resultados}

Escala de prejuicio hacia gais y lesbianas (EPGL)

Como se ha indicado en el apartado de instrumentos, a través de esta escala se obtiene una puntuación por cada sujeto que ha participado que oscilará entre 46 y 184 puntos.

Las puntuaciones obtenidas por los participantes han estado comprendidas entre los 58 y los 94 puntos, siendo la media de 67.90 puntos y una desviación típica de 10.13. Si agrupamos las puntuaciones en función de los cuatro niveles anteriormente descritos, se obtiene lo siguiente:

\begin{tabular}{|lc|}
\hline \multicolumn{2}{|l|}{ Tabla 1. Porcentaje de sujetos y niveles de prejuicio } \\
\hline Nivel & Porcentaje \\
\hline Muy bajo en prejuicios & $0 \%$ \\
Bajo en prejuicios & $67.7 \%$ \\
Alto en prejuicios & $22.6 \%$ \\
Muy alto en prejuicios & $9.7 \%$ \\
\hline
\end{tabular}

Con los resultados podemos afirmar que sigue existiendo un nivel alto y muy alto de prejuicios, así lo indica la puntuación obtenida por más del $30 \%$ de los encuestados.

En cuanto a si existen diferencias entre el sexo de los participantes, la prueba no paramétrica de contrate de hipótesis nos arroja una significación de $p=.20$ por lo que no podemos afirmar que haya diferencias significativas entre hombres y mujeres en cuanto a los prejuicios hacia gais y lesbianas, no obstante, las medias obtenidas muestran que son los varones, con una media de 71.86 puntos (DT=12.07), los que tienen unos prejuicios más altos que las mujeres (Media $=63.73$ y $D T=6.18$ ).

Centrándonos en la variable religión los datos también nos muestran que no existen diferencias significativas $p=.756$ y, además, la media obtenida por los que profesan una religión es muy similar a los que no la profesan (67.95 y 67.75, respectivamente).

Por último, en cuanto a la ideología política, los datos nos muestran que, conforme más a la derecha se situé en el espectro político el sujeto, mayor será la puntuación que obtenga. Así, mientras los de izquierdas obtienen una puntuación media de 63.11 puntos, los de derecha suben la puntuación hasta los 72 puntos. No obstante, tampoco existen diferencias significativas, así lo indica el nivel de significación de .790. 


\begin{tabular}{|lc|}
\hline \multicolumn{2}{|l|}{ Tabla 2. Ideología política y media en la EPGL } \\
\hline Ideología & Media \\
\hline Izquierda & 63.11 \\
Centro-Izquierda & 65.50 \\
Centro & 68.66 \\
Centro-Derecha & 71.25 \\
Derecha & 72.00 \\
\hline
\end{tabular}

Escala de Distancia Social de gais y lesbianas (EDS)

Como se ha especificado en el apartado de instrumentos, con esta escala se puede obtener una puntuación que oscila entre 10 y 50 . Los datos analizados nos relevan que los participantes han obtenido unas puntuaciones que van desde 10 y los 20 puntos, lo que nos muestra que, según los niveles anteriormente citados, se encontrarían en una baja o nula distancia social.

Realizados los cálculos para determinar si existen diferencias significativas entre hombres y mujeres, se ha obtenido un $\mathrm{p}=0.13$ por lo que podemos afirmar que no existen tales diferencias, si bien como en el caso anterior, las mujeres obtienen mejores puntuaciones (Media $=12.29$ y DT=4.26) y los hombres las puntuaciones más altas (Media=16.88 y DT=4.60).

En cuanto a la variable sexo y como ocurriera con la EPGL, en cuanto a la Distancia Social no encontramos diferencias significativas $p=.013$, si bien los datos nos muestran que son las mujeres las que presentan menor distancia social que los hombres, así lo indican las medias obtenidas en esta escala (Media=12.29 y Media=16.88, respectivamente).

En el caso de la religión, nuevamente no encontramos diferencias significativas con un $p=.756$, si bien son los no creyentes (Media=13.50) los que presentan menos distancia social que los que profesan alguna religión (Media=15.11).

Finalmente, en cuanto a la ideología política, nuevamente son los de izquierdas los que presentan una distancia social más baja que los de derechas, si bien no existen diferencias significativas $(p=.790)$.

\begin{tabular}{|ll|}
\hline \multicolumn{2}{|l|}{ Tabla 3. Ideología política y media en la EDS } \\
\hline Ideología & Media \\
\hline Izquierda & 13.54 \\
Centro-Izquierda & 15.00 \\
Centro & 14.91 \\
Centro-Derecha & 15.00 \\
Derecha & 20.00 \\
\hline
\end{tabular}

Interés y formación sobre Diversidad Sexual

En este tercer bloque de resultados se presenta la valoración que los participantes han realizado en torno a las siguientes cuestiones:

- Consciencia de homofobia en los centros educativos

- Interés del alumnado en información y formación sobre diversidad sexual

- Formación recibida en diversidad sexual

- Interés del profesorado por recibir formación sobre diversidad sexual 


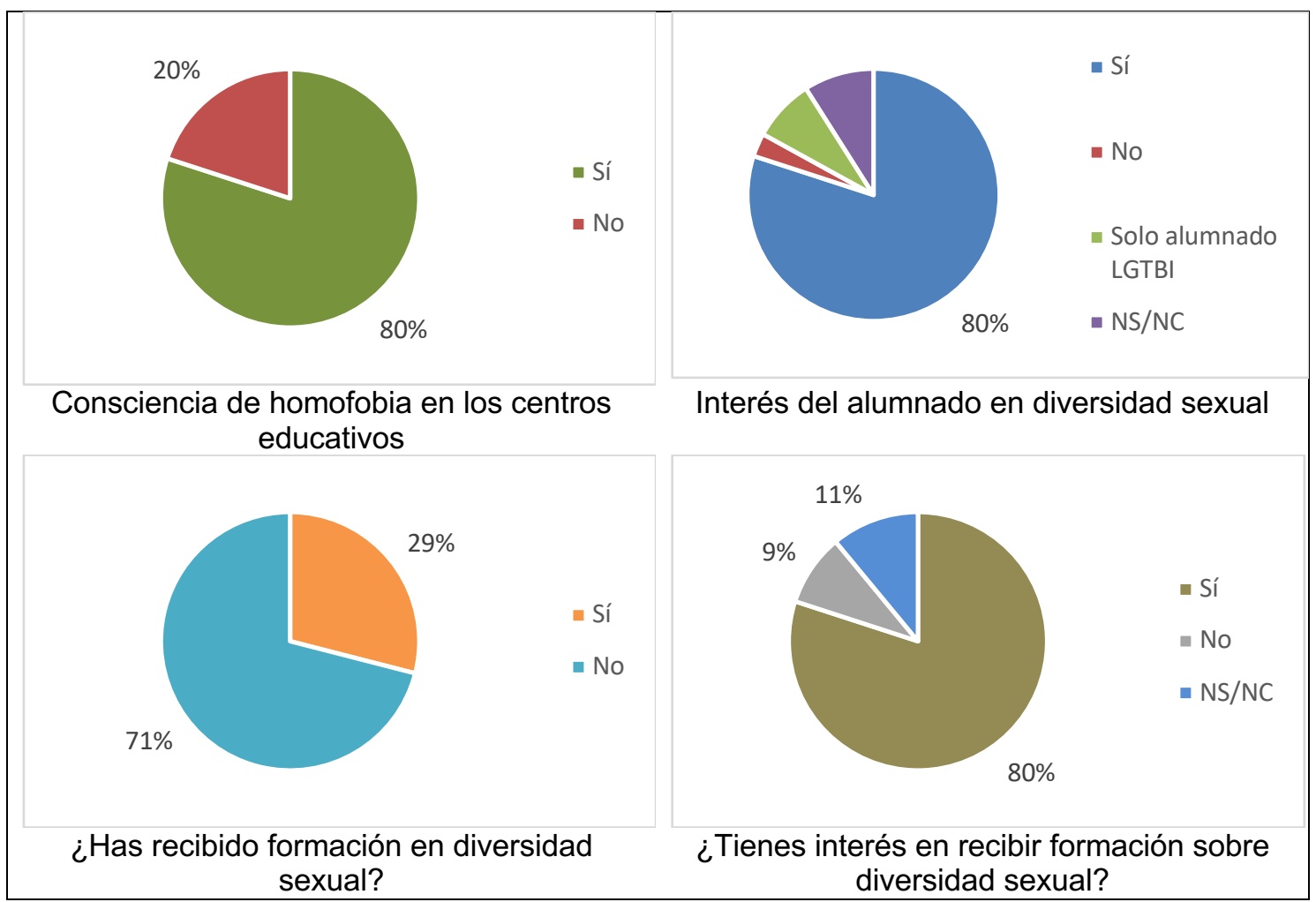

Figura 1. Opinión del alumnado sobre aspectos relacionados con la diversidad sexual

Como se puede observar en la figura anterior, uno de cada cinco docentes no ha percibido que en el centro educativo haya habido casos de homofobia hacia los estudiantes. No obstante, el $80 \%$ de los docentes entienden que los estudiantes mostrarán interés por la formación en torno a esta temática de la diversidad sexual, si bien, llama la atención que haya un pequeño porcentaje (8\%) que estime que esta formación solo la querrán los estudiantes que sean LGBTI. Dos de cada tres docentes $(71 \%)$ informan que no han recibido formación en torno a esta temática. Finalmente, el $80 \%$ muestra interés en recibir formación sobre la diversidad sexual, si bien hay un $9 \%$ que no desea recibir formación en esta temática.

\section{Discusión}

Los resultados que se han expuesto en el apartado anterior son similares a los que se han encontrado en otros estudios. Así pues, uno de cada tres encuestados presenta altos niveles en prejuicios, Esto coincide con los trabajos de Jäckle y Wenzelburger (2015), Rodríguez Otero y Treviño Martínez (2016), Gallardo-Vigil, Segura-Robles y García-Alonso (2017), Robles-Reina, Alemany-Arrebola y Gallardo-Vigil (2017) y Rodríguez Castro y Alonso Ruido (2017). Igualmente, el trabajo de Acuña-Ruiz y Oyuela Vargas (2006) y Cárdenas y Barrientos (2008) que obtienen que las mujeres presentan mejores actitudes que los hombres hacia el colectivo LGTBI. En este sentido, no solo en la EPGL son los varones los que obtienen las puntuaciones más altas, sino que en la EDS ocurre lo mismo, siendo los hombres los que muestran un mayor rechazo a las personas LGTBI.

La religión también afecta a las actitudes y prejuicios hacia las personas gais, lesbianas, transexuales, bisexuales e intersexuales. Aquellos sujetos que profesan una religión obtiene puntuaciones altas tanto en los prejuicios hacia este colectivo como distancia social, frente a los que afirman ser ateos o agnóstico. En este sentido los trabajos de 
Saraç (2015) y de López y Taype-Roldán (2017) establece que la religión es una variable que afecta de forma negativa en la aceptación de las personas LGTBI. De hecho, existe una asociación positiva y significativa entre la religiosidad y el fundamentalismo religioso con respecto a las actitudes negativas hacia los gais y lesbianas (Lazar y Hammer, 2018). Estos mismos datos apuntan los trabajos de Robles-Reina, Alemany-Arrebola y Gallardo Vigil (2017) con estudiantes universitarios entre los que había futuros docentes de secundaria y el trabajo de Sánchez-Herrera (2019).

Por su parte, la ideología política de los encuestados es otra variable que afecta a la forma de entender la diversidad sexual. En un continuo entre la izquierda y la derecha, los datos afirman que son los más conservadores los que presentan unas actitudes y prejuicios más negativos hacia las personas LGTBI (Anderson, Koc y FarlomirPichastor, 2018; Gallardo-Vigil y Rodríguez-Serrano, 2018; Lizana Muñoz, 2009; Morrison y Morrison, 2002).

Los datos también nos muestran que existen docentes que no detectan la homofobia en el aula. Esto puede deberse a que se encuentran normalizados en el día a día algunos insultos y conductas homófobas que pasan desapercibidas para los docentes. Esta falta de detección está muy unida a la falta de formación en temas relacionados con la diversidad sexual (INJUVE, 2011; Pichardo y De Stefano, 2015). Como indica Cornejo Espejo (2018), las situaciones de acoso en el aula por orientación sexual es un tema preocupante por su efecto sobre las víctimas, convirtiendo el centro educativo en un espacio inseguro y que no favorece el aprendizaje ni el desarrollo personal de los alumnos LGTBI. De ahí que sea necesaria la formación específica del profesorado en temas relacionados con la diversidad sexual como apunta Penna (2012).

Podemos concluir, por tanto, que todavía sigue existiendo cierto grado de prejuicio hacia las personas con diversa orientación sexual e identidad de género entre los docentes, principalmente entre los hombres, en los que profesar una religión incide negativamente en las actitudes y prejuicios hacia las personas LGTBI y en la que orientaciones políticas más conservadoras favorecen la aparición de rechazo de aquellos sujetos que se salen de lo heteronormativo.

En este sentido, la existencia de prejuicios hacia los gais y lesbianas influye en la distancia social hacia estas personas. Nuevamente el sexo, ser hombre, la religión, profesora una religión, y la ideología política, ser más conservador, son variables que afectan a la distancia social hacia las personas LGTBI.

Finalmente, se detecta un interés por la formación en temas relacionados con la diversidad sexual, la inexistente en la formación específica en las titulaciones de grado y en el máster de profesorado incide en algunas disfunciones dentro del centro educativo en el que los docentes no perciben los ataques homófobos al identificarlos como simples insultos y por tanto no se incide en la erradicación de estas conductas que afectan a las víctimas de homofobia o LGTBIfobia en el plano personal y en el plano académico. Es necesario, por tanto, comenzar a incluir en la formación de los futuros docentes la temática de la diversidad sexual para un conocimiento de esta realidad social y por tanto presente en nuestras aulas, así como en la formación en estrategias para convertir nuestros centros educativos en centros inclusivos.

Entre las limitaciones del estudio podemos encontrar el bajo número de participantes, solo 35 , si bien debemos mencionar que corresponde a más del $50 \%$ de los docentes del centro en el que se ha realizado la investigación. Se debería intentar aumentar el número de participantes, bien con docentes del mismo centro bien haciendo extensivo el trabajo a otros centros educativos de la ciudad y así ampliar la muestra y poder así 


\section{realizar análisis estadísticos paramétrico en vez de no paramétrico y poder extraer unas conclusiones mucho más amplias.}

\section{Referencias}

Acuña-Ruiz, A.E. \& Oyuela Vargas, R. (2006). Diferencias en los prejuicios frente a la homosexualidad masculina en tres rangos de edad en una muestra de hombres y mujeres heterosexuales. Psicología desde el Caribe, 18, 58-88.

Alemán-Calvache, J. (2018). Historia de vida de un homosexual melillense. Trabajo Fin de Máster. Máster Universitario en Diversidad Cultural: un enfoque multidisciplinar y Transfronterizo. Universidad de Granada, Melilla.

Anderson, J.R.; Koc, Y. y Farlomir-Pichastor, J.M (2018). The English version of attitudes towards homosexuality scale. Swiss Journal of Psichology, 77(3), 1-10.

Cárdenas, M. \& Barrientos, J. (2008). Actitudes explícitas e implícitas hacia los hombres homosexuales en una muestra de estudiantes universitario en Chile. Pykhe, 17(2), 17-25

Chan, C.D. \& Erby, A. (2018). A Critical Analysis and Applied Intersectionality Framework with Intercultural Queer Couples. Journal of homosexality, 65(9), 1249-1274.

Cornejo Espejo, J. (2018). Discriminación y violencia homofóbica en el sistema escolar: estrategias de prevención, manejo y combate. Revista Barsileira de Educação, 23, 1-24.

Francisco Amat, A.; Aguirre García-Carpintero, A. y Moliner Miravet, L. (2018). Heterosexual, ¿qué es eso? Percepciones sobre identidades sexuales en educación secundaria. Revista de Investigación Educativa, 36(1), 93-108.

Gallardo-Vigil, M.A. y Rodríguez-Serrano, M. (2018). Actitudes hacia la diversidad sexual en un contexto multicultural. En 6th International Congress of Educational Sciences and Development. Setúbal (Portugal), Del 21 al 23 de junio.

Gallardo-Vigil, M.A.; Rojas-Ruiz, G. y López-Vallejo, M.A. (2019). Actitudes hacia la diversidad sexual. El respeto a las diferencias. En MA. Gallardo-Vigil y I. Alemany-Arrebola, Las actitudes ante diversas realidades sociales. Buenas prácticas para la educación inclusiva (pp. 83-101). Granada: Editorial Comares.

Gallardo-Vigil, M.A.; Segura-Robles, A. y García-Alonso, M. (2017). Investigaciones sobre actitudes y Diversidad Cultural en el Campus de Melilla de la Universidad de Granada hacia diversas problemáticas sociales. En 5 th International Congress of Educational Sciences and Development. Santander, del 25 al 37 de mayo.

García, H. (1984). Actitudes hacia los homosexuales y las lesbianas de cuatro grupos de estudiantes universitarios. Tesis de maestría, Departamento de Psicología. Universidad de Puerto Rico.

INJUVE. (2011). Jóvenes y diversidad sexual. Madrid: INJUVE. Recuperado de: http://www.injuve.es/observatorio/salud-ysexualidad/jovenes-y-diversidad-sexual

Jäckle, S. \& Wenzelburger, G. (2015). Religion, Religiosity, and the Attitudes Toward Homosexuality-A Multilevel Analysis of 79 Countries. Journal of Homosexuality, 62(3), 207-241.
Lazar, A., \& Hammer, JH. (2018). Religiousness and antigay/lesbian attitudes: The mediating function of intratextual religious fundamentalism. Psychology of Violence, 8(6), 763771.

Ley 13/2005, de 1 de julio, por la que se modifica el Código Civil en materia de derecho a contraer matrimonio. Boletín Oficial del Estado, núm. 157, de 2 de julio de 2005. Recuperado de: https://www.boe.es/buscar/doc.php?id=BOE-A-2005-11364

Ley $3 / 2007$, de 15 de marzo, reguladora de la rectificación registral de la mención relativa al sexo de las personas. Boletín Oficial del Estado, núm, 65, de 16 de marzo de 2007. Recuperado de: https://www.boe.es/buscar/pdf/2007/BOE-A-2007-5585consolidado.pdf

Lizana Muñoz, V.A. (2009). Representaciones sociales sobre heterosexualidad y homosexualidad de los/las estudiantes de Pedagogía en los contextos de formación docente inicial. Estudios Pedagógicos, 35(1), 117-138.

López, C.E. y Taype-Roldan, A. (2017). Asociación entre compromiso religioso y presentar actitudes negativas hacia hombres homosexuales, en un grupo de estudiantes de medicina peruanos. Acta Médica Peruana, 34(1), 33-40.

Morrison, M. A. \& Morrison T. G. (2002). Development and validation of a scale measuring modern prejudice toward gay men and lesbian women. Journal of Homosexuality, 43, 15-37.

Penna, M. (2012). Formación del profesorado en la atención a la diversidad afectivo-sexual. Tesis Doctoral, Universidad Complutense de Madrid. Recuperada de: http://eprints.ucm.es/16718/1/T34011.pdf

Pew Reserah Center (2013). The global divide on homosexuality. Recuperado de: https://www.pewresearch.org/global/2013/06/04/the-globaldivide-on-homosexuality/

Pichardo, J.I., \& De Stefano (2015). Abrazar la diversidad: propuestas para una educación libre de acoso homofóbico y transfóbico. Madrid: INMUJER.

Robles-Reina, R., Alemany-Arrebola, I. y Gallardo-Vigil, M.A. (2017). Actitudes prejuiciosas hacia las personas homosexuales en estudiantes universitarios en Melilla. MODULEMA. Revista científica sobre diversidad cultural, 1 , 165-186.

Rodríguez Otero, L.M. y Treviño Martínez, L. (2016). Sexismo y actitudes hacia la homosexualidad, la bisexualidad y la transexualidad en estudiantes de Trabajo Social mexicanos. Trabajo Social Global, 6(1), 3-30.

Rodríguez-Castro, Y. y Alonso-Ruido, P. (2017). Evaluación de las actitudes hacia la diversidad sexual de adolescentes del ámbito semi-rural gallego. Revista de estudios e Investigación en Psicología y Educación, volumen extraordinario n. ${ }^{\circ}$ 2, 5-9. 
Russell, J. (2013). Whose Better? (re)Orientating a Queer Ecopedagogy. Canadian Journal of Environmental Education, $19,11-26$.

Sánchez Herrera, C. (2018). Comparativa de las actitudes hacia el colectivo LGTBI en las fuerzas armadas y en la población civil. MODULEMA. Revista científica sobre diversidad cultural, 3 , $45-62$

Saraç, L. (2015). Relationships between religiosity level and attitudes toward lesbians and gay men among Turkish university students. Journal of Homosexuality, 62(4), 481-494.

Toro-Alfonso, J., \& Varas-Díaz, N. (2004). Los otros: prejuicio y Distancia Social hacia hombres gay y lesbianas en una muestra de estudiantes de nivel universitario. International Journal of Clinical and Health Psychology, 4(3), 537-551.

Vázquez-Rivera, M.; Caleb, E. y Toro Alfonso, J. (2018). Hacia una psicología libre de paños tibios: actitudes, prejuicios y distancia social de psicoterapeutas hacia gays y lesbianas. Perspectivas en Psicología: Revista de Psicología y Ciencias Afines, 15(1), 15-24. 